\title{
SOME CRITERIA FOR NILPOTENCY IN GROUPS AND LIE ALGEBRAS
}

\author{
EUGENE SCHENKMAN ${ }^{1}$
}

We shall say that an automorphism $\alpha$ is nilpotent or acts nilpotently on a group $G$ if in the holomorph $H=[G](\alpha)$ of $G$ with $\alpha, \alpha$ is a bounded left Engel element, that is, $[H, k \alpha]=1$ for some natural number $k$. Here $[H, k \alpha]$ means $[H,(k-1) \alpha]$ with $[H, 0 \alpha]$ denoting $H$.

Let $G^{\prime}$ denote the commutator subgroup $[G, G]$, and let $\Phi(G)$ denote the Frattini subgroup of $G$. If $\alpha$ is an automorphism of a nilpotent group $G$ such that the automorphism $\bar{\alpha}$ induced by $\alpha$ on $G / G^{\prime}$ is nolpotent (or with certain restrictions on the exponent of $G$ on $G / \Phi(G)$ ), then by a well-known theorem of Philip Hall (cf. [6, p. $202]), \alpha$ is nilpotent. Here we shall show that the same conclusion follows if we know that the restriction of $\alpha$ to a suitable subgroup of a nilpotent group is nilpotent. We prove the following two theorems announced in [7].

THEOREM 1. Let $G$ be a nilpotent group, let $\alpha$ be an automorphism of $G$, let $F$ be a subgroup of $G$ stable under $\alpha$ and such that $\alpha$ is nilpotent on $F$. If $F$ contains its centralizer $C_{G}(F)$, then $\alpha$ is nilpotent on $G$.

For the statement of Theorem 2 it will be convenient to say that a nilpotent group $G$ is of height $k$ if $k$ is the least nonnegative integer so that for each prime $p$ and each $p$-element $g$ of $G, g^{p^{k}} \in G^{\prime}$.

THEOREM 2. Let $G$ be a nilpotent group of height $k$, let $\alpha$ be an automorphism of $G$, let $F$ be a subgroup of $G$ stable under $\alpha$ and such that $\alpha$ is nilpotent on $F$. Suppose that $F$ contains the elements of order 4 of $C_{G}(F)$, the elements of order $p$ of $C_{G}(F)$ for all odd primes $p$, and the torsion-free elements of $C_{G}(F)$. Then $\alpha$ is nilpotent on $G$.

Theorem 2 includes as a special case a recent result of Blackburn (cf. [1]).

In view of the known results about Engel elements we have the following consequence.

COROLLARY 1. Let $F$ be a nilpotent normal subgroup of a group $G$ and suppose that $G$ is either finite or else solvable with nilpotent HirschPlotkin radical $H$, and suppose further that either $F \geqq C_{G}(F)$ or that $F$

Received by the editors March 12, 1968 and, in revised form, August 19, 1968.

1 The author is indebted to the National Science Foundation for support. 
is as in Theorem 2. If for each $x \in G$, the inner automorphism $\alpha_{x}$ determined by $x$ induces a nilpotent automorphism on $F$, then $G$ is nilpotent.

Since Theorem 1 is very closely related to a theorem of Thompson (cf. [3, p. 185]) we include the following generalization of the latter.

THEOREM 3. A nilpotent group $G$ of finite height has a characteristic subgroup $C$ with the following properties:

(i) $C / Z(C)$ has height at most one provided $G$ is periodic.

(ii) $[G, C]$ is contained in the center $Z(C)$ of $C$ (and hence $C$ has class at most two).

(iii) $C_{G}(C)=Z(C)$.

(iv) Every nonnilpotent automorphism of $G$ induces a nonnilpotent automorphism of $C$.

We also develop similar ideas for Lie algebras as follows. We say that a derivation $\delta$ is nilpotent or acts nilpotently on a Lie algebra $L$ if in the holomorph $H=L+\{\delta\}$ of $L$ with $\delta, \delta$ is an Engel element. Then we have for Lie algebras the following analogues to our results for groups:

THEOREM 4. Let $F$ be a subalgebra of a nilpotent Lie algebra $L$ such that $F$ contains its centralizer $C_{L}(F)$. If $\delta$ is a derivation of $L$ which maps $F$ onto $F$ and is nilpotent on $F$ then $\delta$ is nilpotent on $L$.

CoRollary 2. Let $F$ be an ideal of a finite-dimensional Lie algebra $L$ such that $F$ contains $C_{L}(F)$. If for each $x$ in $L$ the inner derivation $\delta_{x}$ determined by $x$ induces a nilpotent derivation on $F$, then $L$ is nilpotent.

Theorem 5. A nilpotent Lie algebra L has a characteristic subalgebra $C$ with the following properties:

(i) $[L, C]$ is contained in the center $Z(C)$ of $C$ and hence $C$ has class at most two.

(ii) $C_{L}(C)=Z(C)$.

(iii) Every nonnilpotent derivation of $L$ induces a nonnilpotent derivation of $C$.

Proofs. We note that easy induction arguments immediately give the following:

(1) The holomorph of a nilpotent group with a nilpotent automorphism is nilpotent.

(2) A nilpotent automorphism of a nilpotent group of finite exponent (of exponent $p^{k}$ ) has finite order (of order a power of $p$ ).

(3) If $G$ has a normal series $G=G_{0}>G_{1}>\cdots>G_{n}=1$ and an automorphism $\alpha$ so that for $i=1,2, \cdots, h, \alpha$ maps $G_{i}$ onto $G_{i}$ and 
if $\alpha$ is nilpotent on each factor group $G_{i-1} / G_{i}$, then $\alpha$ is nilpotent on $G$.

Proof of Theorem 1 . We consider the case first where $G^{\prime} \leqq F$. Since $[F, k G]=1$ and $[F, m \alpha]=1$ for appropriate natural numbers $k$ and $m$, it follows that $F$ has a normal series $F=F_{0}>F_{1}>\cdots>F_{n}=1$ so that if $H$ denotes the holomorph $[G](\alpha)$ then $\left[F_{i}, H\right] \leqq F_{i+1}$ for $i=0,1, \cdots, n-1$. Let $C_{i}$ denote $C_{G}\left(F_{i}\right)$. Then $C_{0} \leqq C_{1} \leqq \cdots \leqq C_{n}$ $=G$ and $C_{0} \leqq F$. If $C_{r+1}$ is the least of the $C_{i}$ not in $F$ we shall show that $\alpha$ is nilpotent on $C_{r+1}$ as follows: $\left[F_{r}, C_{r+1}\right] \leqq F_{r}$ so that $\left[\left[F_{r}, C_{r+1}\right], m \alpha\right]$ $=1$. Then for $c \in C_{r+1}, f \in F_{r}$,

$$
\left[\left[f, c^{-1}\right], \alpha\right]^{c}\left[\left[c, \alpha^{-1}\right], f\right]^{\alpha}\left[\left[\alpha, f^{-1}\right], c\right]^{f}=1
$$

(cf. $\left(^{*}\right)$, p. 201 of $\left.[6]\right)$, and hence $\left[\left[f, c^{-1}\right], \alpha\right]=\left[f,\left[c, \alpha^{-1}\right]\right]^{\alpha}$ $=\left[f^{\alpha},[\alpha, c]\right]$. It follows that $\left[\left[\left[f, c^{-1}\right], \alpha\right], \alpha\right]=\left[\left[f^{\alpha},[\alpha, c]\right], \alpha\right]$ $=\left[f^{\alpha^{2}},[\alpha,[c, \alpha]]\right]$ and $\left[\left[f, c^{-1}\right], j \alpha\right]=\left[f^{\alpha i},[\alpha,[c,(j-1) \alpha]]\right]$ for each $j>1$. Since $\left[\left[F_{r}, C_{r+1}\right], m \alpha\right]=1$ it follows that $\left[C_{r+1}, m \alpha\right] \leqq C_{r} \leqq F$ and $\left[C_{r+1}, 2 m \alpha\right]=1$. Hence $\alpha$ is nilpotent on $C_{r+1}$ and consequently on $F C_{r+1}$. An induction then gives that $\alpha$ is nilpotent on $C_{n}=G$ and the statement of the theorem is proved in the case where $G^{\prime} \leqq F$. Now let $G^{2}$ denote $G^{\prime}$ and for $t>2$, let $G^{t}$ denote $\left[G^{t-1}, G\right]$ so that $G>G^{2}>\ldots$ $>1$. In the general case suppose that $G^{t}$ is the least member of the lower central series not in $F$. Then $\alpha$ is nilpotent on $F G^{t}$ by what was shown above and an induction gives that $\alpha$ is nilpotent on $G$. This proves Theorem 1.

Proof of Theorem 2. Let $G=G_{0}>G_{1}>\cdots>G_{n}=1$ be an invariant series of $G$ which includes the members of the lower central series of $G$ and so that each factor $G_{i} / G_{i+1}$ has height at most 1. Let $r$ be maximal so that $G_{r+1} \leqq F$ and assume inductively that the theorem is true for all $s<r$. Now $F \cap C_{G}(F)$ is central in $C_{G}(F)$ and the hypotheses of the theorem hold for $F \cap C_{G}(F)$ in $C_{G}(F)$. If we can show that $\alpha$ is nilpotent on $C_{G}(F)$, then by (3) $\alpha$ will be nilpotent on $F C_{G}(F)$, and by Theorem $1, \alpha$ will be nilpotent on $G$.

Accordingly we need only consider the case where $F$ is central in $G$. Then all the torsion free elements of $G$ are in $F$ and we let $k$ be maximal so that $F$ contains all the elements of order $p^{k}$ of $G$ for all $p(k>0$ by hypothesis). Let $c$ be a $p$-element of $G_{r}$ for some $p$; since $\alpha$ is nilpotent on $F$, a suitable $p$ th power $\beta$ of $\alpha$ is the identity on the Sylow $p$-subgroup $F_{p}$ of $F$ by (2). Then $[c, \beta]^{p k}=\left(c^{-1} c^{\beta}\right)^{p^{k}}$ and since $\left[c^{-1}, c^{\beta}\right]$ is in $G_{r}$, hence in $F$ and therefore central,

$$
\left(c^{-1} c^{\beta}\right)^{p^{k}}=c^{-p^{k}} c^{\beta p^{k}}\left[c^{-1}, c^{\beta}\right]^{C_{p k, 2}}
$$

(where $C_{p k, 2}$ is the binomial coefficient), which is $c^{-p k} c^{\beta_{p} k}$ since

$$
\left[c^{-1}, c^{\beta}\right]^{c_{p k, 2}}=\left[c^{-p}, c^{\beta}\right]^{c_{p} k, 2 / p}=1
$$


(for $k=1$ it is only necessary to consider odd $p$ ). Thus $[c, \beta]^{p k}=\left[c^{p k}, \beta\right]$ $=1$, and hence $[c, \beta] \in F$. Since $\beta$ is a $p$ th power of $\alpha$ it follows that $\alpha$ (modulo the centralizer of $c$ in $(\alpha)$ ) and $c$ generates a $p$-subgroup whose order is bounded in terms of the class of nilpotency and height of $G$ independent of the element $c$. Thus $\alpha$ is nilpotent on $F_{p} \cap G_{r}$. Since this is true for each $p, \alpha$ is nilpotent on $G_{r}$ and hence by (3) on $F G_{r}$. By the induction assumption $\alpha$ is nilpotent on $G$ and the theorem is proved.

Proof of Corollary 1. Suppose first that $G$ is finite. An induction argument on order gives that all maximal subgroups of $G$ containing $F$ are nilpotent. Hence $G / F$ is solvable and thus $G$ is solvable. We now consider the case where $G$ is solvable with nilpotent HirschPlotkin radical $H$ and consider the subgroups $F \leqq H \leqq K \leqq G$ where $K$ is a normal subgroup of $G$ with $K / H$ abelian. Then for $x \in K, \alpha_{x}$ is nilpotent on $H$ by the theorems and hence $x$ is a left Engel element of $G$. It follows from Theorem 4 of [4] that $x$ is in $H$ and therefore $K \leqq H$; since $G$ is solvable it follows that $G \leqq H$ and hence that $G$ is nilpotent, as was to be shown.

Proof of Theorem 3. Theorem 1 includes the implication that condition (iii) of Theorem 3 implies condition (iv). Accordingly we need only prove that conditions (i), (ii), and (iii) hold for $G$. We let $D$ be a maximal characteristic abelian subgroup of $G$ and let its centralizer $C_{G}(D)$ be denoted by $H$. We let $K$ be the complete inverse image of the maximal subgroup of height one of the center of $G / D$ when $G$ is periodic, while for $G$ not periodic $K$ will be the complete inverse image of the center of $G / D$ (so that $[G, K] \leqq D$ ). We then let $C$ be $H \cap K$ and $Q$ be $C_{G}(C)$, noting that $Q \leqq H$ since $D \leqq C$. Since all the above subgroups are characteristic in $G, D(C \cap Q)$ is characteristic as well as abelian, so that from the maximality of $D$ it follows that $C \cap Q \leqq D$ and therefore $H \cap K \cap Q=K \cap Q \leqq D$. Furthermore, since $C \leqq C_{G}(D)$, it follows that $D$ is in $C$ and from the maximality of $D$ that $D$ is in fact the center of $C$. Finally the fact that $K \cap Q \leqq D$ implies that $Q \leqq D$; for in the contrary case, modulo $D, Q$ would be a nontrivial normal subgroup which did not meet the maximal subgroup of height one of the center of $G$ (modulo $D$ ). Thus conditions (i), (ii), and (iii) are satisfied for $C$ and the proof of the theorem is complete. It is worthy of notice that in case $C_{G}(D) \leqq D$, then $D$ itself satisfies the conditions of the theorem in place of $C$.

Proofs of Theorems 4 And 5. The proof of Theorem 4 is essentially the same as that of Theorem 1 except that the argument to replace the lines following $\left(^{*}\right)$ is less complicated, since the Jacobi identity is similar to but simpler than $\left(^{*}\right)$. For the proof of Theorem 5 we let $D$ be a maximal characteristic subalgebra and let $K$ be the 
complete inverse image of the center of $L / D$ and then proceed as in Theorem 3. It should be remarked here that in a similar fashion the arguments on p. 202 of [6] for groups can be recast directly to give the analogous results for Lie algebras (cf. [2]).

Proof of Corollary 2. $L$ induces a nilpotent algebra of linear transformations on the vector space $F / F^{\prime}$ and hence by Engel's theorem (cf. [5] for instance) $L / F$ is nilpotent. Thus $L$ is solvable. By an induction argument $F L^{\prime}$ is nilpotent and hence by Theorem 4 every $x \notin L F^{\prime}$ is an Engel element of $L$. Then by Engel's theorem again, $L$ is nilpotent.

\section{REFERENCES}

1. N. Blackburn, Automorphisms of finite p-groups, J. Algebra 3 (1966), 28-29.

2. C. Y. Chao, Some characterizations of nilpotent Lie algebras, Math. Z. 103 (1967), 40-42.

3. D. Gorenstein, Finite groups, Harper and Row, New York, 1968.

4. K. W. Gruenberg, The Engel elements of a solvable group, Illinois J. Algebra 3 (1959), 151-168.

5. N. Jacobson, Lie algebras, Interscience, New York, 1962.

6. E. Schenkman, Group theory, Van Nostrand, Princeton, N. J., 1965.

7. —, Nilpotent automorphisms, Notices Amer. Math. Soc. 13 (1966), 501.

Purdue University 